\title{
Gas explosion analysis of safety gap effect on the innovating FLNG vessel with a cylindrical platform
}

\author{
Jingde $\mathrm{Li}^{1, \text { a }}$, Guowei Ma ${ }^{2, \mathrm{~b}}$ Hong Hao ${ }^{1, \mathrm{c}}$, Yimiao Huang ${ }^{2, \mathrm{~d}}$ \\ ${ }^{1}$ Centre for Infrastructural Monitoring and Protection, School of Civil and \\ Mechanical Engineering, Curtin University, Kent Street, Bentley, WA 6102, \\ Australia \\ ${ }^{2}$ School of Civil, Environmental and Mining Engineering, the University of \\ Western Australia, 35 Stirling Highway, Crawley WA 6009, Australia \\ ajingde.li@curtin.edu.au, bma@civil.uwa.edu.au, chong.hao@curtin.edu.au, \\ d20442592@student.uwa.edu.au
}

\begin{abstract}
After investigating gas dispersion on a cylindrical Floating Liquefied Natural Gas (FLNG) platform [1], this second article focuses on assessment of gas explosion by using Computational Fluid Dynamics (CFD). Gas explosion simulations are carried out to evaluate the explosion overpressure mitigating effect of safety gap. The Datadump technique, which is an effective tool in resetting turbulence length scale in gas explosion overpressure calculation, is applied to ensure simulation accuracy for the congestion scenario with safety gap. Two sets of different safety gaps are designed to investigate the safety gap on the cylindrical FLNG platform, the overall results indicate that the safety gap is effective in reducing overpressure in two adjacent congestions. However, for the explosion scenario where the flame is propagating through several safety gaps to the far field congestion, the safety gap mitigates overpressure only in certain explosion protecting targets. Two series of artificial configurations are modelled to further investigate the explosion scenarios with more than two safety gaps in one direction. It is concluded that the optimal safety gap
\end{abstract}


design in overpressure mitigation for the cylindrical FLNG platform is to balance the safety gap distance ratio in the congested regions.

Keywords: gas explosion; safety gap; FLACS; cylindrical FLNG; CFD

\section{Introduction}

Presently, engineers have been constructing increasingly more offshore projects in less accessible fields in more challenging environments, such as the far deeper waters and the fields in the arctic weather. Meanwhile, some companies at the forefront of technology in the oil and gas industry have been developing some new and improved solutions to operate the oil and gas drilling and production in the marginal fields.

The Floating vessels for Liquefied Nature Gas (FLNG) and Floating Production Storage and Offloading (FPSO) are good examples of the new engineering technologies $[2,3]$, which replace the fixed offshore platforms and pipeline facilities to become the better choice for deep water oil and gas exploration. Along with the advantage of the storage ability, these FLNG and FPSO vessels have also the flexibility to be anchored and towed out at different locations in the ocean $[4,5]$.

However, most current FLNGs are designed as ship-shaped vessels that can only drift in one direction. When extreme weather conditions such as strong wind and waves occur, ship-shaped vessels cannot provide a stable platform due to the great hull deflection caused by its long ship body. Hence, during a typhoon, the wind and waves could lead to a maritime catastrophe. In addition, the mooring structure - turret is required in the design of the ship-shaped FLNG to mitigate the effect of 
weathervaning, and the overall cost due to the construction of a turret consequently increases.

In order to improve the stability of the FLNGs, the cylindrical shape platforms are conceptually designed. The symmetrically designed FLNGs not only diminish the fatigue loads induced by wave, but can also face the environment with same shape in all directions. For instance, in the arctic fields, the cylindrical shape vessels have same ice-breaking features for all ice drift directions, whereas the ship-shaped ones are onedirectionally operable. Economically, the need for cost-driving turret and swivel systems is eliminated in the circular hull design [6]. Therefore, the cylindrical solution, which is based on the conventional ship-shaped FLNG, provides a significant improvement in hydrodynamic stability and cost-effectiveness.

However, regarding the development and research of cylindrical platforms, the work done so far focused on construction, operation studies, hydraulic and hydrodynamic analysis[2, 7-9], while the safety evaluations for the circular vessels subjected to gas explosion have been in an empty field. Additionally, the existing vessels with cylindrical platforms worldwide are mainly on-duty for crude oil drilling and production, whereas those cylindrical-hulled FLNG designed by researchers and engineers are still in the conceptual phase, and without gas explosion safety evaluations. Following up the authors' previous investigation of the gas dispersion on the cylindrical FLNG [1], the aim of this paper is to perform the gas explosion safety analysis on the obstructed configurations with different sets of safety gaps. In this 
study, a cylindrical FLNG platform configuration, which is composed of 12 liquefaction unit modules with detailed equipment and piping design, is established and used in the gas explosion simulations. Moreover, the gas explosion mitigating effect of safety gap on the cylindrical FLNG platform is investigated

The safety gap which is an open space, with no congestion, deliberately placed in between congested process areas, is one of the most effective and widely used safety measures. The principle behind the operation of the safety gap is that it basically interrupts a positive feedback mechanism in congested areas. The positive feedback mechanism consists of the generation of turbulence, enhanced thermal and chemical mixing between combustion products and reactants, higher flame speeds and thereby both higher pressures and even higher turbulence intensities and so on. The absence of obstacles in a safety gap eliminates the fluid- obstacle interaction thereby preventing the generation of turbulence [10].

In this study, a series of gas explosions are performed on the cylindrical FLNG platform in order to analyze the safety gap effect on overpressure mitigation. The CFD based software FLACS[11], which is a strongly validated finite volume N-S solver tool [12-15] and has been developed continuously for over 40 years for consequence prediction of gas ventilation, dispersion and explosion, has been utilized in this study. In order to improve the FLACS overpressure calculation accuracy, the Data-dump technique proposed in previous work [10] is also employed. 


\section{Numerical models}

Based on the prototype of the ship-shaped FLNG vessel as seen in Fig. 1, The cylindrical FLNG platform in this paper is modeled by using FLACS, all anticipated congestion - walls and decks are assumed to be rigid during the CFD simulations[11]. Volume Block Ratio (VBR) within a given zone in the model, is defined as the ratio between the total volume of the geometrical objects in that zone and the total volume of the zone. The homogeneous cubical grid cells are applied as the grid models in the explosion simulations.

\subsection{Ship-shaped FLNG}

The PRICO [16-18] FLNG units are used in this study. A realistic ship-shaped FLNG model including liquefaction trains, dehydration and mercury removal modules and compressors, etc. is established in detail as shown in Fig. 1.

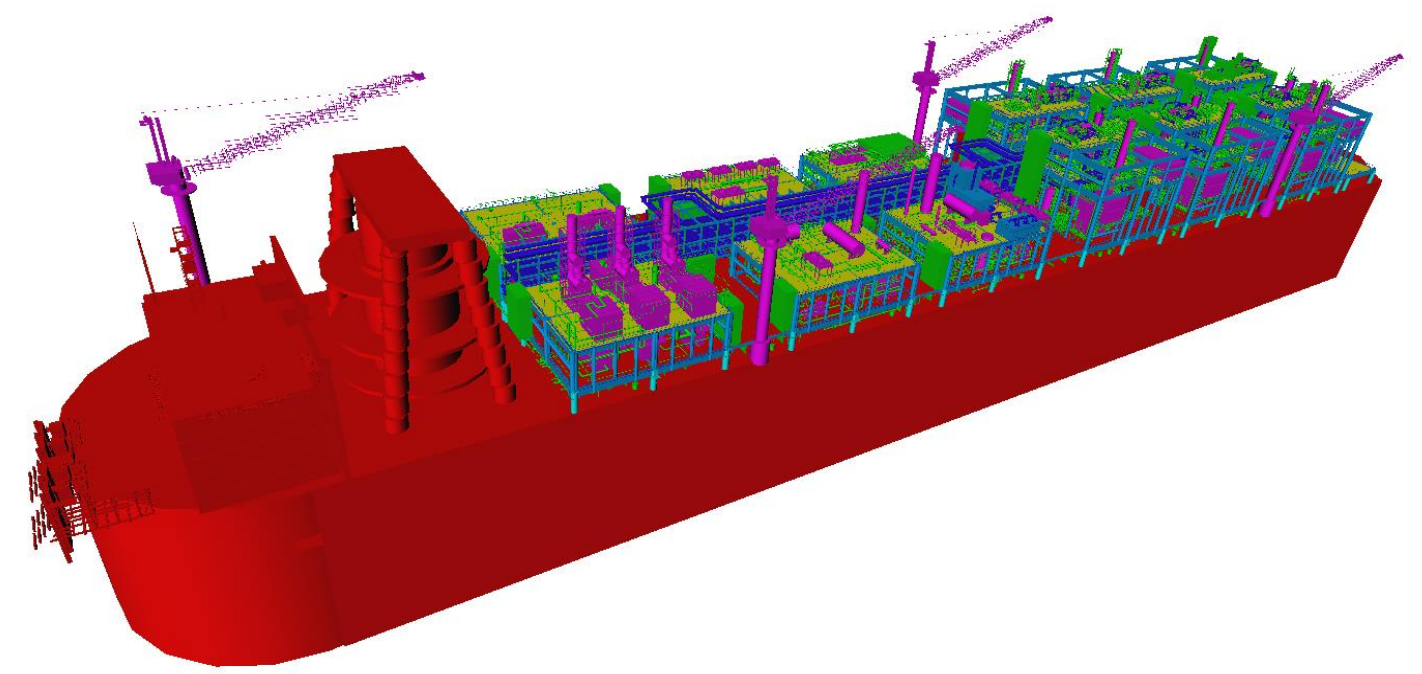

Fig. 1 The ship-shaped FLNG 3D geometry in FLACS 


\subsection{Cylindrical FLNG}

As shown in Fig. 2, the three dimensional cylindrical FLNG platform is modeled based on the ship-shaped FLNG. The order of the 12 modules on the cylindrical FLNG platform is kept the same as that in the ship-shaped FLNG, and the modules are organized in a $U$ shape to fit the cylindrical hull. Consequently, the cylindrical FLNG platform has a more compact area with 12 modules in the same process order and the turret area is eliminated. One of the liquefaction trains- Module 7 is shown in a closer view in Fig. 3. As seen in Fig. 4, the topside modules on the platform include:

1. Power generation (Module 1)

2. 3 Trent gas turbines and 2 essential diesel generators (Module 2)

3. Nitrogen package, hot oil, Mono-Ethylene-Glycol (MEG) processing and inlet facilities (Module 3)

4. Boil off gas compressor and fuel gas system (Module 4)

5. Acid gas removal unit \& end flash gas compressor (Module 5)

6. Dehydration and mercury removal (Module 6)

7. Liquefaction modules (Module 7 to Module 12) 


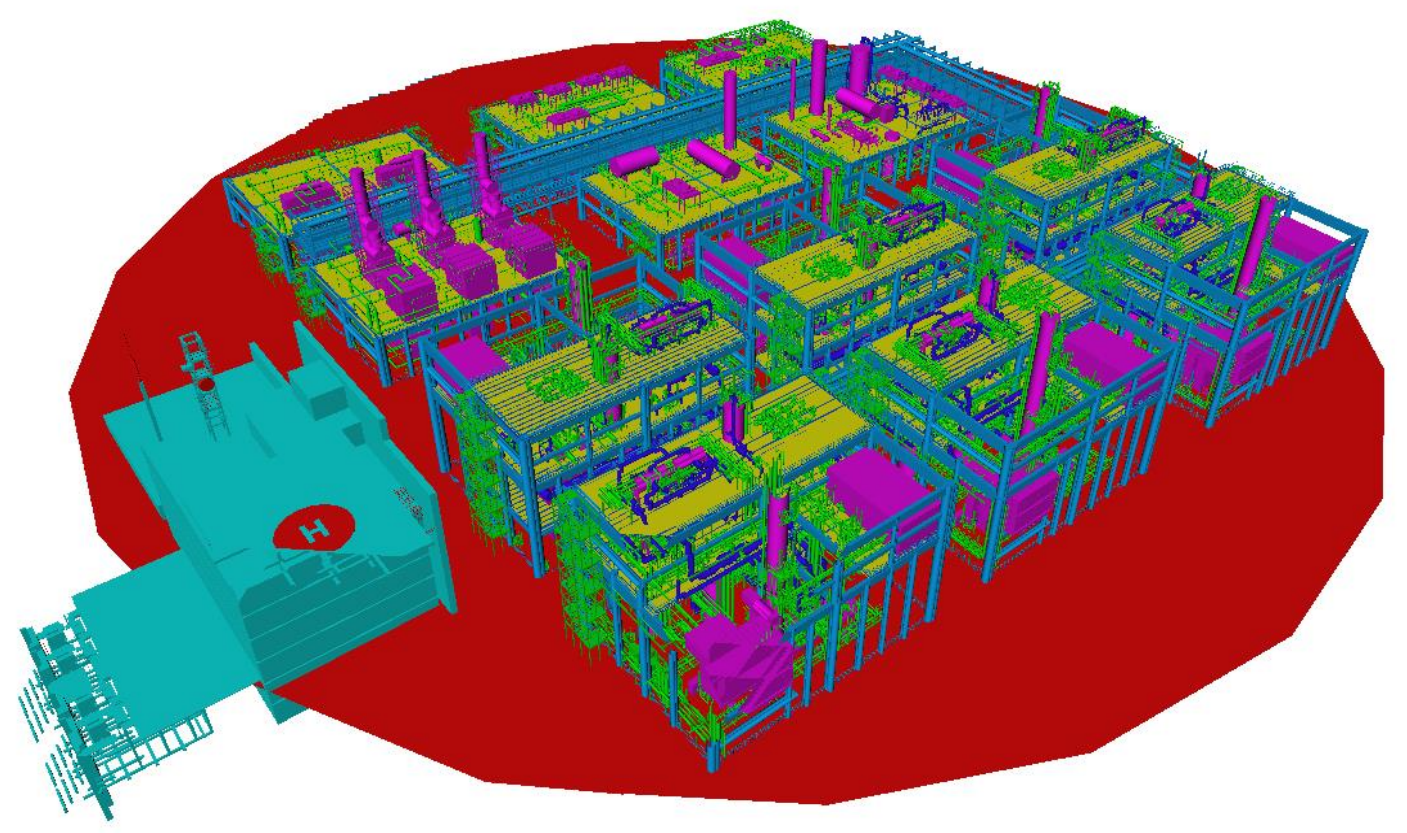

Fig. 2 FLACS geometry of the cylindrical FLNG platform

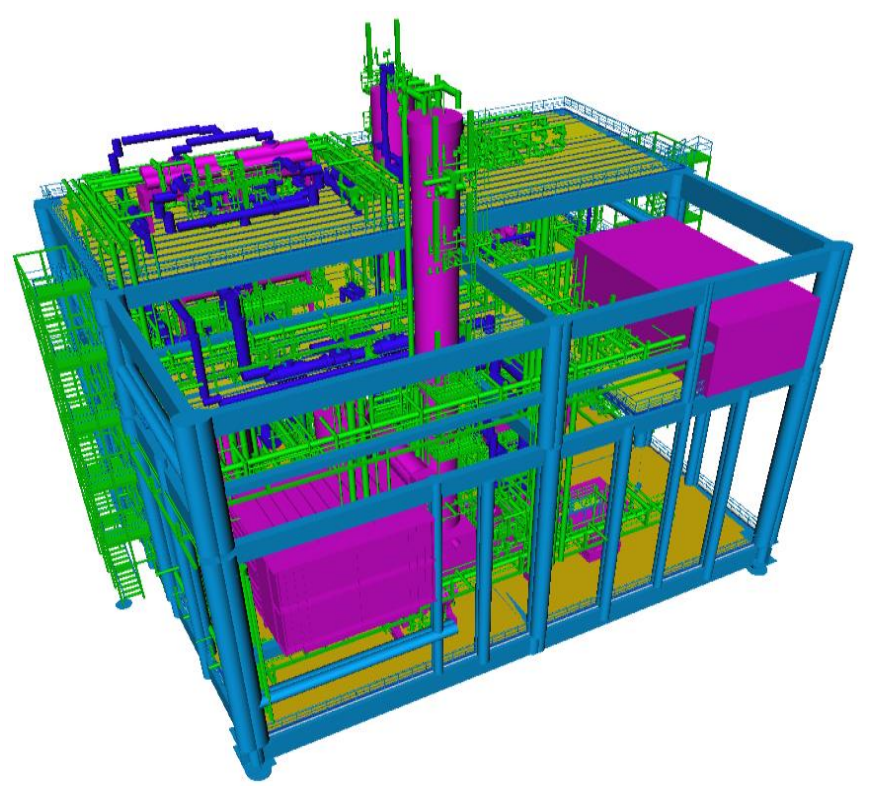

Fig. 3 A close view of the liquefaction train 


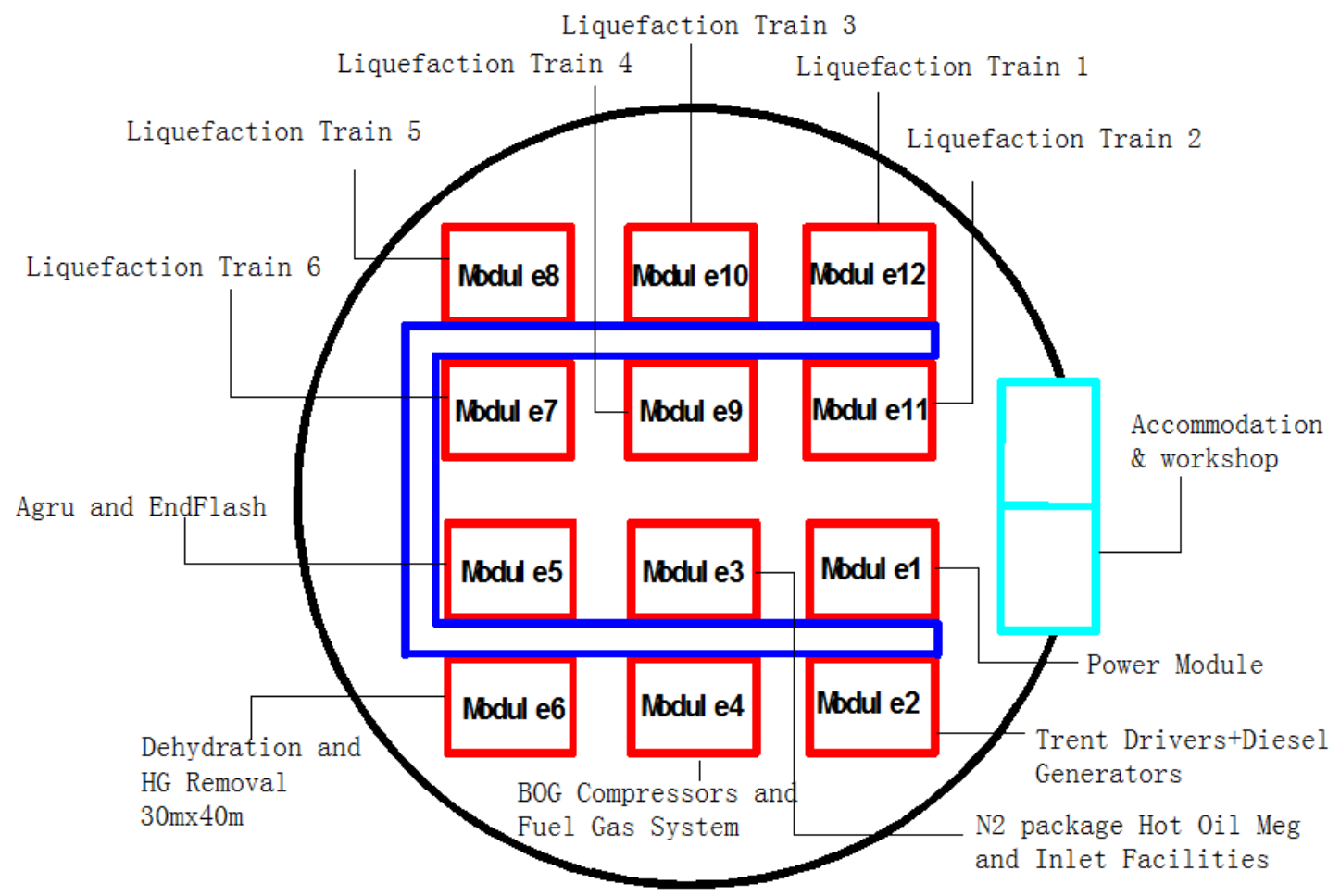

Fig. 4 Topside arrangement of the modules on the cylindrical FLNG platform

\section{Safety gap effect on gas explosion}

A series of gas explosion scenarios are then simulated on the cylindrical FLNG platform. The effects of different safety gaps on the platform are investigated in both near field and far field explosion regions. The Data-dump technique is utilized to improve the calculation accuracy of FLAC.

\subsection{Near field gas explosion simulation on the cylindrical FLNG platform}

On the cylindrical FLNG platform, the authors define the near field gas explosion region as the scenario where flames propagate through two adjacent congestions with one safety gap. Two different safety gaps of $12.5 \mathrm{~m}$ and $20 \mathrm{~m}$ are modeled as seen in Fig. 5. Furthermore, Fig. 6 shows the major equipment in the liquefaction module 
which includes the turbine air intake, turbine bundle removal equipment, scrubber and cold box on the lower level and two heat exchangers on the top levels. The detectors are placed on the lower level to monitor overpressures, the gas composition is $27 \%$ Methane, 33\% Ethane, 15\% propane, 19\% Pentane and 6\% Nitrogen.

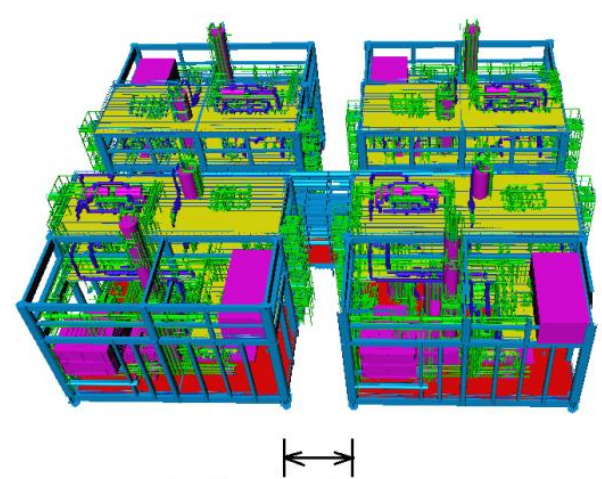

12. $5 \mathrm{~m}$ safety gap

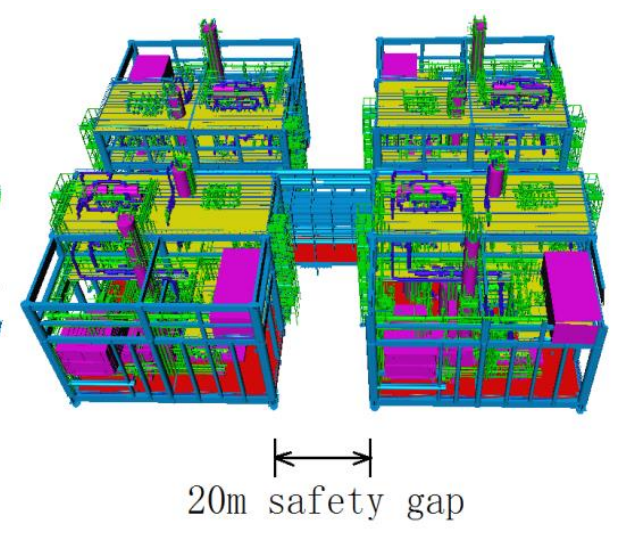

$20 \mathrm{~m}$ safety gap

Fig. 5 Two configurations with different safety gaps in near field

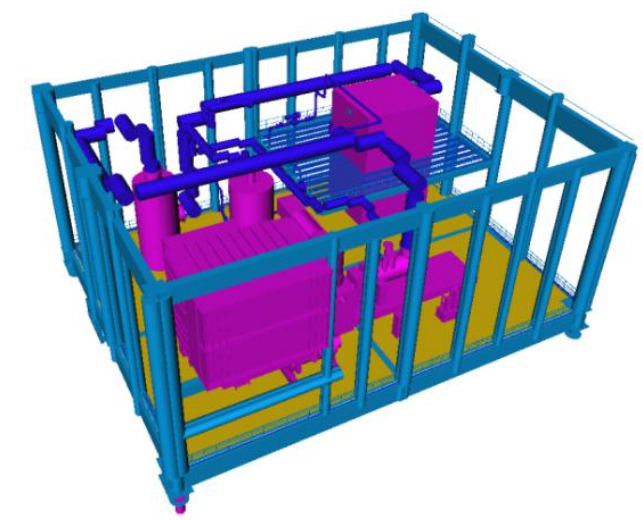

(a) 3D view of the liquefaction lower level

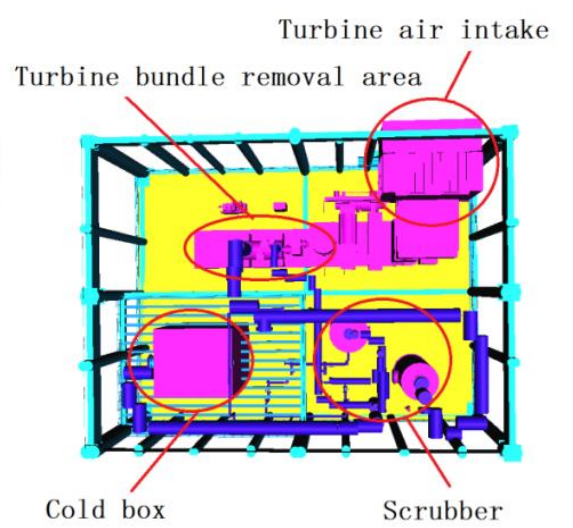

(b) Monitor regions

Fig. 6 Lower level of the liquefaction train

\subsubsection{Application of Data-dump in gas explosion simulations on the cylindrical FLNG platform}

Before the comparison of different sets of the safety gaps, the Data-dump technique [10] is firstly applied in this study. The Data-dump is an effective tool to reset the turbulence length scale for gas explosion scenario with a safety gap, thereby increasing calculation accuracy of FLACS. In this study, the 25\%, 50\% and $100 \%$ 
filled gas cloud cases are all investigated, the overpressures before and after Datadump are monitored under or on the surfaces of the turbine air intake, turbine bundle removal equipment, scrubber and cold box, as seen in Table 1.

Table 1 Overpressures before and after Data-dump technique

\begin{tabular}{|c|c|c|c|c|c|}
\hline \multirow{2}{*}{$\begin{array}{c}\text { Gas } \\
\text { cloud } \\
\text { coverage }\end{array}$} & \multirow[b]{2}{*}{ Monitor region } & \multicolumn{2}{|c|}{ 20m safety gap } & \multicolumn{2}{|c|}{$12.5 \mathrm{~m}$ safety gap } \\
\hline & & $\begin{array}{l}\text { Overpressure } \\
\text { before Data- } \\
\text { dump (bar) }\end{array}$ & $\begin{array}{l}\text { Overpressure } \\
\text { after Data- } \\
\text { dump (bar) }\end{array}$ & $\begin{array}{l}\text { Overpressure } \\
\text { before Data- } \\
\text { dump (bar) }\end{array}$ & $\begin{array}{c}\text { Overpressure } \\
\text { after Data- } \\
\text { dump (bar) }\end{array}$ \\
\hline \multirow{4}{*}{$\begin{array}{l}100 \% \\
\text { filled }\end{array}$} & Turb air intake & 7 & 6.4 & 10.5 & 10 \\
\hline & Turb bun removal & 3.6 & 2.6 & 4.1 & 4 \\
\hline & Cold box & 2.8 & 2.5 & 3.2 & 3.1 \\
\hline & Scrubber & 6.2 & 5 & 11.5 & 10.5 \\
\hline \multirow{4}{*}{$\begin{array}{l}50 \% \\
\text { filled }\end{array}$} & Turb air intake & 6.5 & 6.4 & 9.2 & 9.1 \\
\hline & Turb bun removal & 3.1 & 2.9 & 3.8 & 3.7 \\
\hline & Cold box & 2.5 & 2 & 2.8 & 2.6 \\
\hline & Scrubber & 5.8 & 4.5 & 10.5 & 9.2 \\
\hline \multirow{4}{*}{$\begin{array}{l}25 \% \\
\text { filled }\end{array}$} & Turb air intake & 3.4 & 2.6 & 4.3 & 3.8 \\
\hline & Turb bun removal & 2.1 & 2 & 2.3 & 2.2 \\
\hline & Cold box & 0.29 & 0.28 & 0.9 & 0.8 \\
\hline & Scrubber & 1.65 & 1.1 & 5 & 3.9 \\
\hline
\end{tabular}

For each gas explosion simulation, the explosion results are initially dumped at the time when the flame exits the edge of the donor which is the module where the gas cloud is ignited. Then by creating a cc-file and executing a duplication command, a new explosion file for the receiving module is created along with the data loaded from overpressure results in the donor. In order to reset the turbulence length scale and restart the flame acceleration in the receiving module, the ignition is relocated to the upstream edge of the accepting module which is opposite to the donor.

The comparison data of the overpressure modification percentages due to Data-dump technique are depicted in Fig. 7 and Fig. 8. Overall, the Data-dump technique 
decreases the overpressures recorded in the acceptor module. Specifically, for the $12.5 \mathrm{~m}$ safety gap scenario, the overpressure modification percentages tend to be below $12 \%$ except for the overpressures observed in the scrubber area. Whereas the overpressures are modified to larger extents in the $20 \mathrm{~m}$ safety gap case, the percentage for each case in Fig. 8 is greater than the corresponding case in Fig. 7. In other words, the greater the safety gap size is, the more over-prediction of overpressure in FLACS should be amended by Data-dump technique. Therefore, in order to assure the overpressure calculation accuracy in safety gap modeling, the Data-dump is applied for all the following simulations in this study.

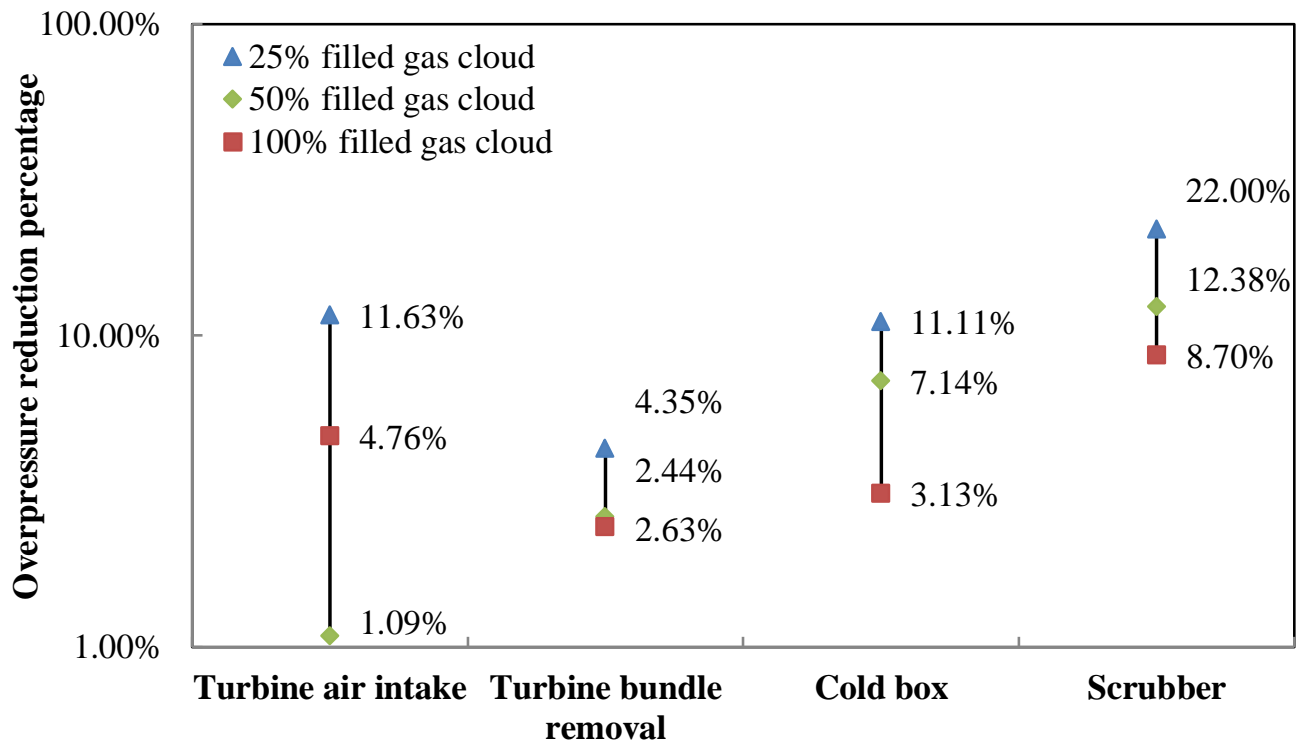

Fig. 7 Overpressure reduction percentages after Data-dump for $12.5 \mathrm{~m}$ safety gap scenario with different gas cloud coverage 


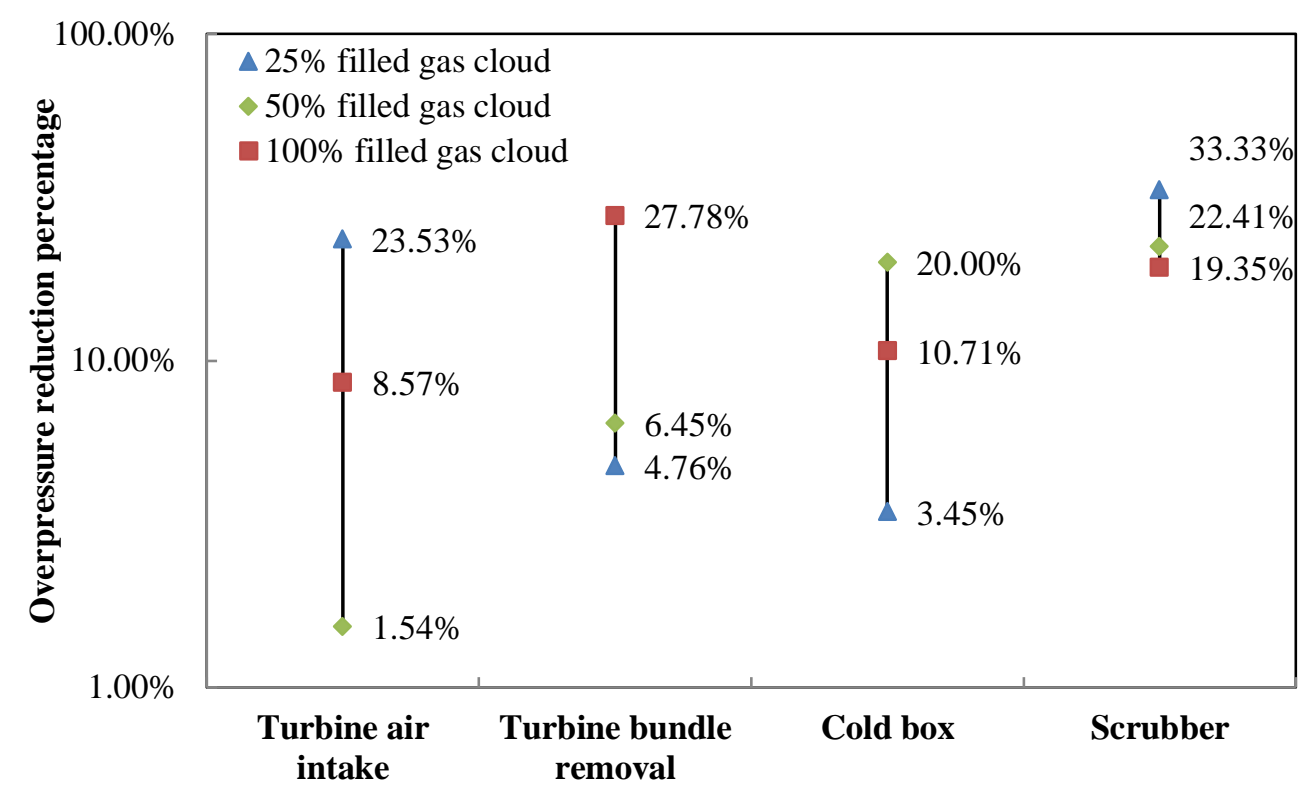

Fig. 8 Overpressure reduction percentages after Data-dump for $20 \mathrm{~m}$ safety gap scenario with different gas cloud coverage

\subsubsection{Different safety gaps subjected to gas explosion under different gas cloud coverage}

After the application of Data-dump into the gas explosion overpressure calculation in FLACS, the safety gap effect on overpressure mitigation is then investigated in the adjacent (near field) modules - liquefaction trains. Two sets of safety gap (12.5m and 20m) configurations are modelled, Fig. 9 gives an overview of the $100 \%$ gas filled simulation case with the maximum overpressure up to 9 bar. The results indicate that nearly identical pressures are observed in the donor modules (the modules where ignition occurs on the left hand side of the safety gap) for both of $20 \mathrm{~m}$ gap and $12.5 \mathrm{~m}$ gap configurations. However, in the comparison between the $20 \mathrm{~m}$ gap and $12.5 \mathrm{~m}$ gap cases, it is seen that pressures are lower in the receiving module (modules on the other side) in the $20 \mathrm{~m}$ gap case than that in the $12.5 \mathrm{~m}$ gap case. 


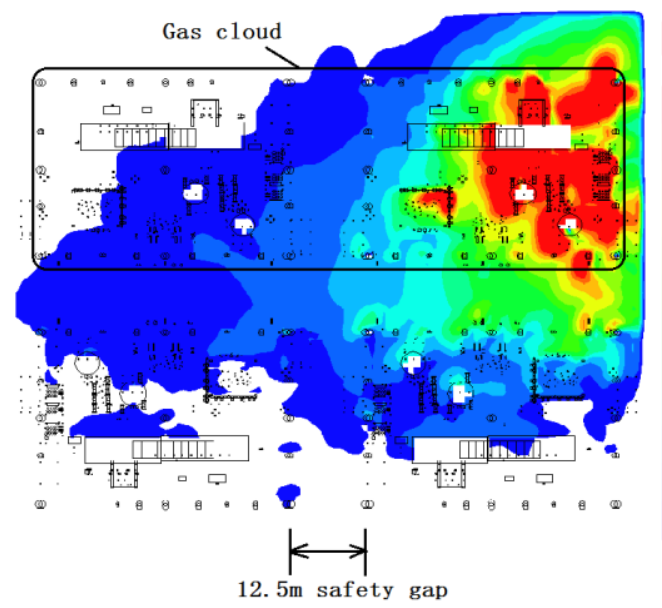

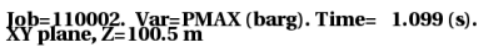

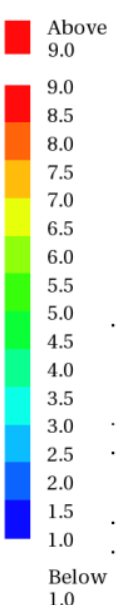

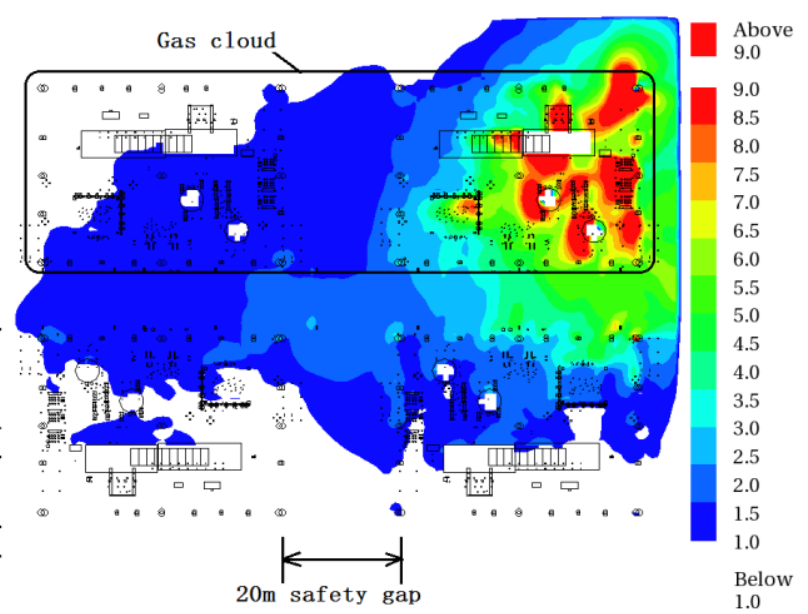

KYb=110001. $\operatorname{Var}=\operatorname{PMAX}($ barg) $\mathrm{Pime}=1.100(\mathrm{~s})$.

(a) Simulation case of $12.5 \mathrm{~m}$ safety gap

(b) Simulation case of $20 \mathrm{~m}$ safety gap Fig. 9 Maximum overpressures for $100 \%$ filled gas cloud spanning the modules separated by different safety gaps

In addition, the comparison of all the $25 \%, 50 \%$ and $100 \%$ gas cloud filled cases is conducted, and the coverage percentage is controlled by manipulating the volume height of the gas cloud. Fig. 10 to Fig. 12 illustrate three similar scenarios where a reduction in the overpressure is observed in the receiving modules if the safety gap increases from $12.5 \mathrm{~m}$ to $20 \mathrm{~m}$.

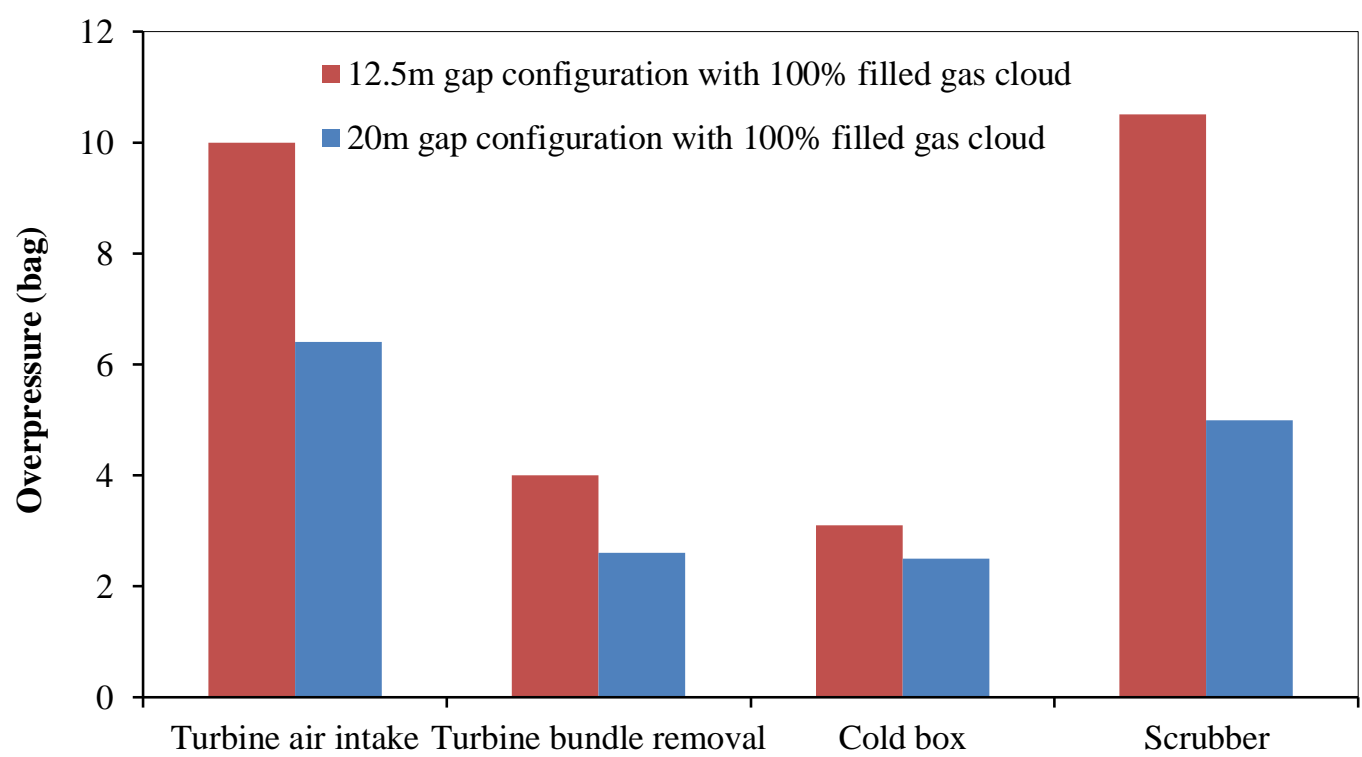

Fig. 10 Maximum overpressures for cloud (100\% filling) configurations with $12.5 \mathrm{~m}$ and $20 \mathrm{~m}$ safety gaps 


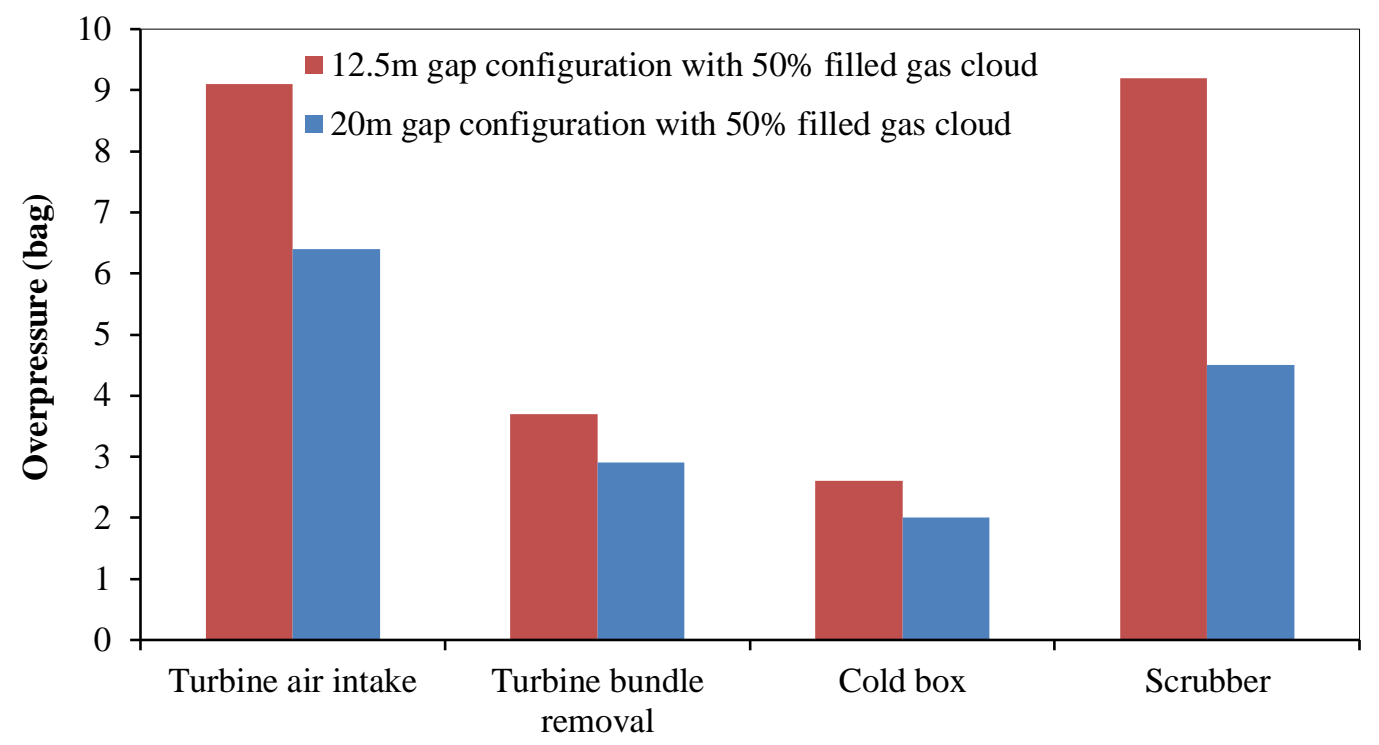

Fig. 11 Maximum overpressures for cloud (50\% filling) configurations with $12.5 \mathrm{~m}$ and $20 \mathrm{~m}$ safety gaps

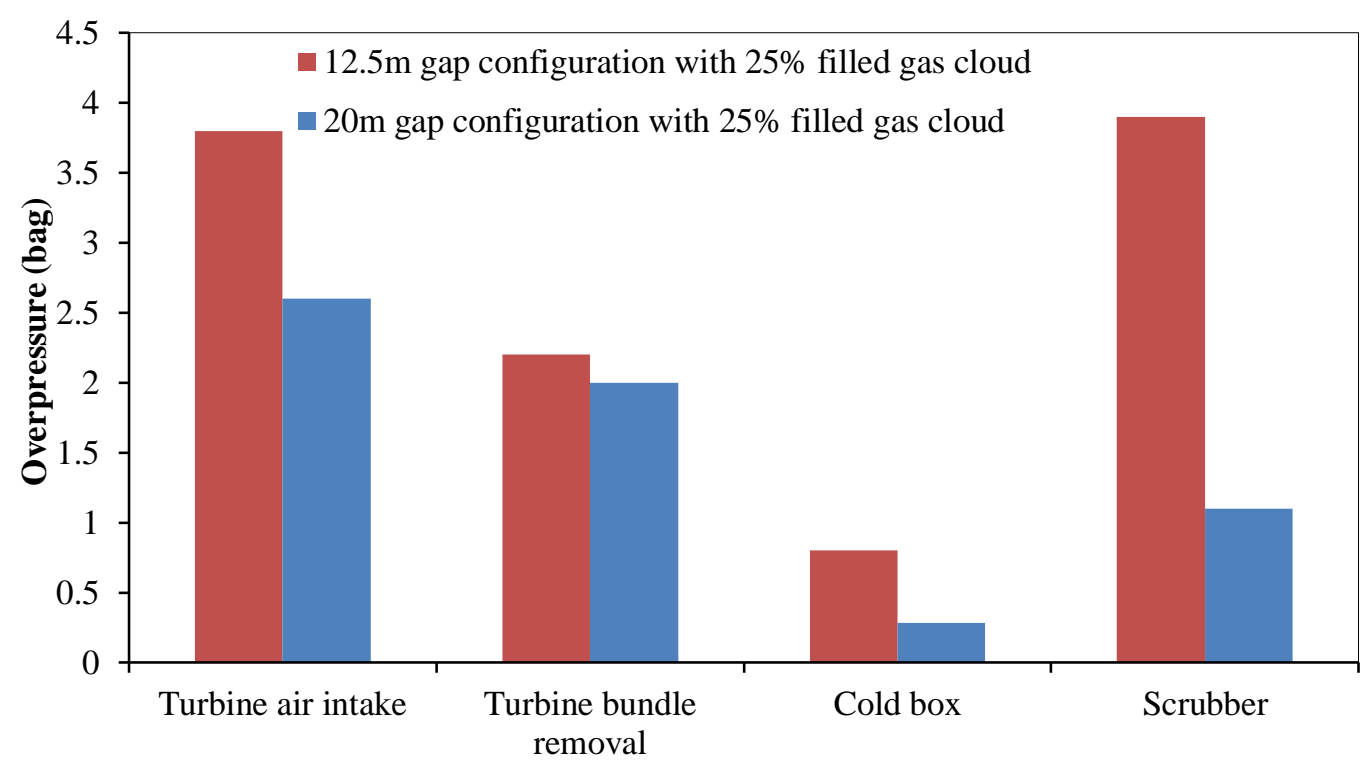

Fig. 12 Maximum overpressures for cloud (25\% filling) configurations with $12.5 \mathrm{~m}$ and $20 \mathrm{~m}$ safety gaps

It is interesting to note that overpressures near the turbine air intake and scrubber are higher than the observed overpressures in the cold box and turbine bundle removal areas regardless of the safety gap size. The main reason is that the flame propagation distances from the ignition in the donor to the targets of the turbine air intake and scrubber are longer than the flame propagation distances to the cold box and turbine 
bundle removal areas (as seen in Fig. 6(b), where the turbine air intake and scrubber are placed on the right hand side even further away from the ignition coming from left). The longer the flame path length is, the longer time the flame turbulence develops within the congestion, which further induces greater overpressures $[19,20]$. Moreover, the turbine air intake and scrubber region are more congested with small dimension objects. Therefore, the smaller average diameter of the obstacles and greater congestion ratio contribute to more turbulence induced flame acceleration, which builds up greater overpressures as well [21, 22].

In terms of the safety gap effect, it is seen that the overpressure difference between the $12.5 \mathrm{~m}$ and $20 \mathrm{~m}$ safety gap cases is more apparent in the turbine air intake and scrubber areas, which indicates that the safety gap reduces more overpressures where the flame path is longer and the average obstacle diameter is smaller and the congestion ratio is greater. In addition to the analysis of the safety gap effect on overpressure mitigation in near field, the investigation to the safety gap effect in far field is further conducted below.

\subsection{Far field gas explosion simulation on the cylindrical FLNG platform}

In order to investigate the consequence associated with the ignition of gas cloud from one module to the far away modules on the cylindrical platform, explosion simulations are performed by using varying parameters such as different gas cloud locations, size and ignition locations. The far field gas explosion scenario is defined as the region where flame propagates through more than one safety gap. 4 gas clouds 
with same size of $140 \times 140 \times 10 \mathrm{~m}$ are placed at different locations covering all the modules, for each gas cloud, 6 ignition scenarios occurring in the ground center of each module are simulated, as seen in Fig. 13. Overall, for each cylindrical FLNG platform, 24 simulations are carried out in the far field gas explosion investigation.

And two different cylindrical FLNG platforms (as seen in Fig. 14) are modeled in order to compare the effect of different safety gaps on gas explosion mitigation. One configuration is the platform with all modules moved $10 \mathrm{~m}$ inwards within the pipe rack circle to form the safety gap of 10m in North-South direction (Fig. 14(b)), while the other configuration has no gap between the modules and pipe rack, as seen in Fig. 14(a).

Fig. 14 shows one explosion scenario where the gas cloud is ignited in Module 10 and the flame propagates further to all other surrounding modules and far field ones such as Module 3 and Module 4. The gas explosion path going through two gaps in NorthSouth direction is defined as Path 1, e.g. Path 1 in Fig. 14 is distance from Module 10 to Module 3 or from Module 9 to Module 4, whereas Path 2 is defined as the flame path after three gaps. For each module, about 10 monitor points are uniformly allocated on the ground level to detect the overall overpressures. The recorded overpressures tabulated in Table 2 are averaged from the modules in the far end of the flame path. Table 2 and Fig. 15 illustrate the comparison results observed on the cylindrical FLNG platform with two different safety gap setups. 


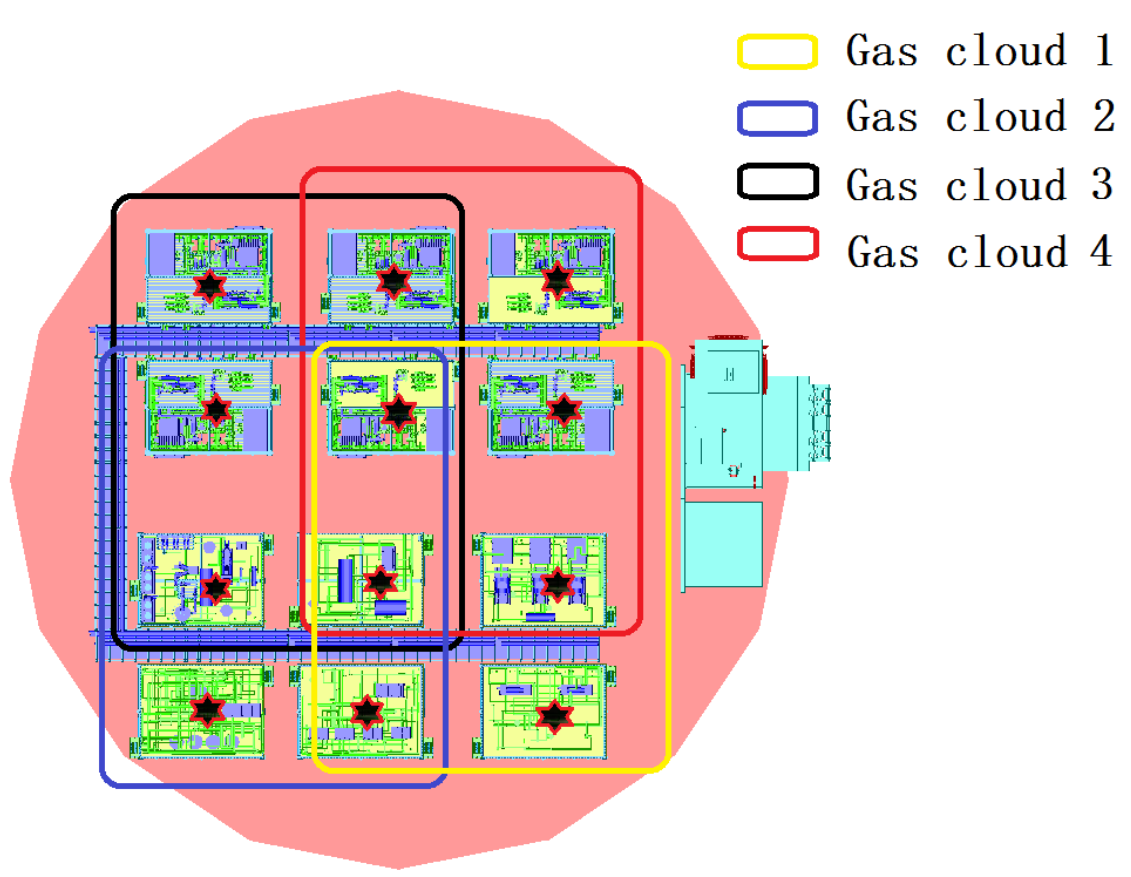

Fig. 13 Overview of explosion scenarios ignited in each module
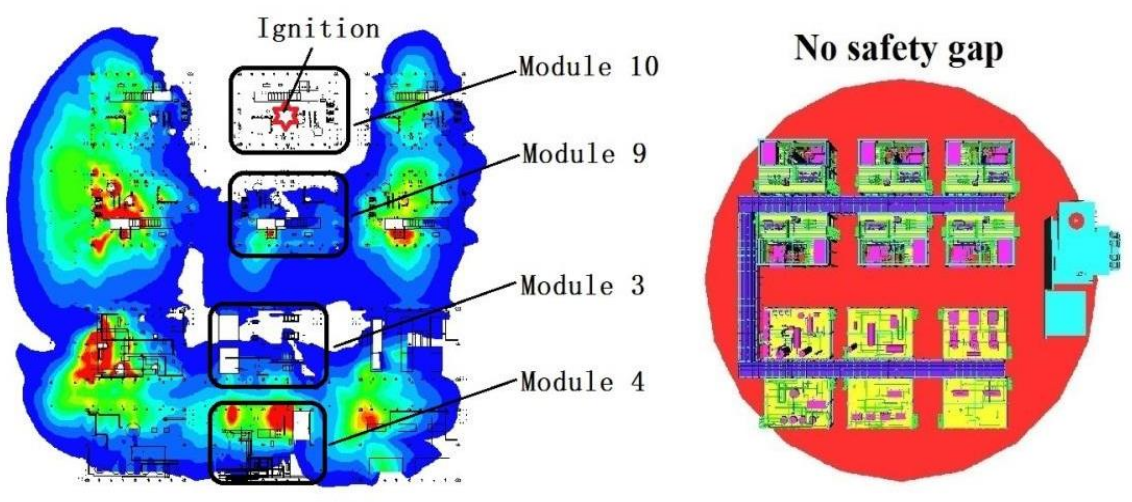

(a) Modules in the cylindrical FLNG without gap

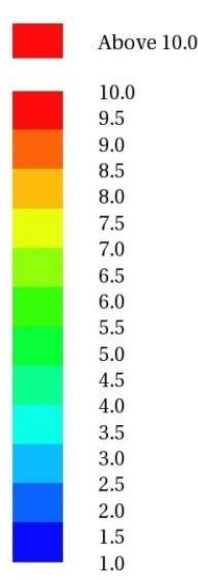

Below 1.0 

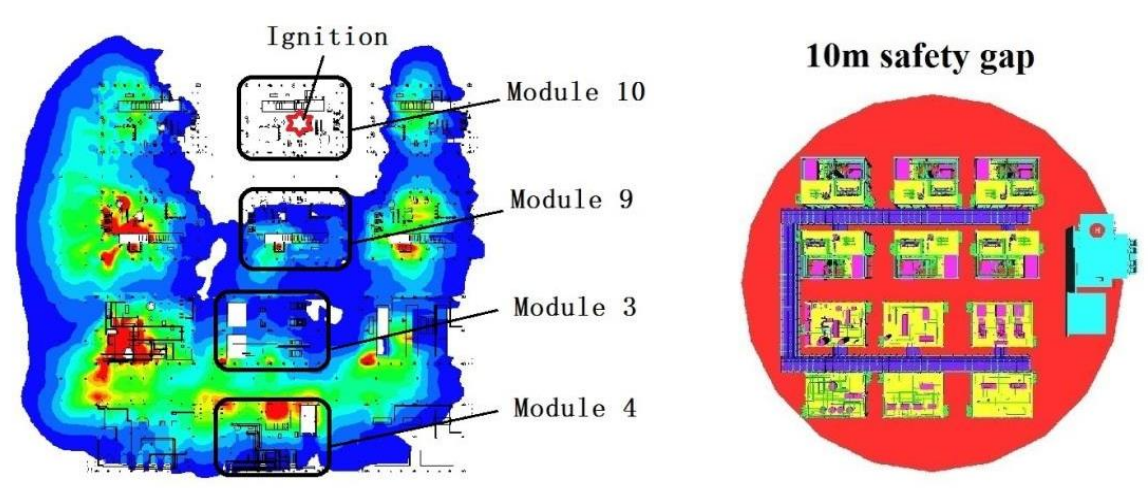

(b) Modules in the cylindrical FLNG with $10 \mathrm{~m}$ gap

Fig. 14 Test layout of the cylindrical FLNG for gas cloud ignited in Module 10

Table 1 Results of the far field gas explosion simulations

\begin{tabular}{|c|c|c|c|c|}
\hline Case no. & Ignition location & Gas cloud no. & $\begin{array}{l}\text { Overpressure without } \\
\text { safety gap }\end{array}$ & $\begin{array}{l}\text { Overpressure with } \\
10 \mathrm{~m} \text { safety gap }\end{array}$ \\
\hline \multicolumn{5}{|c|}{ Flame propagation path 1} \\
\hline 1 & Module 1 & 4 & 4.11 & 3.84 \\
\hline 2 & Module 3 & 4 & 3.96 & 3.72 \\
\hline 3 & Module 3 & 3 & 5.00 & 4.71 \\
\hline 4 & Module 5 & 3 & 5.77 & 5.48 \\
\hline 5 & Module 7 & 2 & 6.55 & 6.47 \\
\hline 6 & Module 9 & 2 & 8.04 & 7.68 \\
\hline 7 & Module 9 & 1 & 5.17 & 4.79 \\
\hline 8 & Module 11 & 1 & 3.90 & 3.68 \\
\hline 9 & Module 12 & 4 & 1.47 & 1.89 \\
\hline 10 & Module 10 & 4 & 1.47 & 2.04 \\
\hline 11 & Module 10 & 3 & 3.52 & 4.48 \\
\hline 12 & Module 8 & 3 & 3.80 & 5.07 \\
\hline 13 & Module 6 & 2 & 3.51 & 4.43 \\
\hline 14 & Module 4 & 2 & 3.14 & 4.01 \\
\hline 15 & Module 4 & 1 & 2.15 & 2.97 \\
\hline 16 & Module 2 & 1 & 1.98 & 2.66 \\
\hline \multicolumn{5}{|c|}{ Flame propagation path 2} \\
\hline 17 & Module 2 & 1 & 2.30 & 2.22 \\
\hline 18 & Module 4 & 1 & 2.47 & 2.03 \\
\hline 19 & Module 4 & 2 & 3.06 & 2.31 \\
\hline 20 & Module 6 & 2 & 1.72 & 1.67 \\
\hline 21 & Module 8 & 3 & 2.04 & 1.78 \\
\hline 22 & Module 10 & 3 & 2.37 & 1.97 \\
\hline 23 & Module 10 & 4 & 1.80 & 1.62 \\
\hline 24 & Module 12 & 4 & 2.18 & 1.76 \\
\hline
\end{tabular}




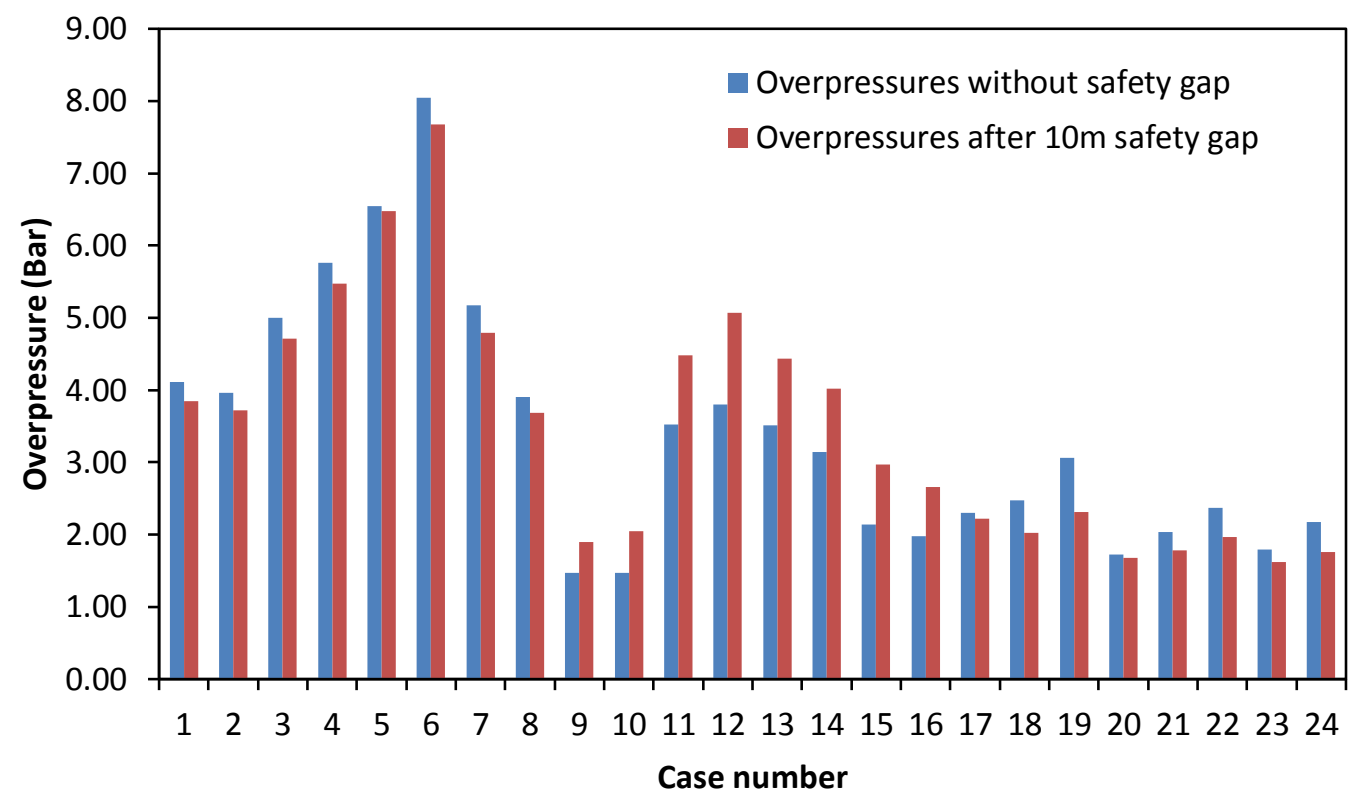

Fig. 15 Overpressures recorded in the far end of the flame

For gas explosion flame propagating through path 1, it is seen in Fig. 15 that the overpressures between the two FLNG layouts have two distinct group results. Precisely, for simulation group case 1 to 8 , the gas explosion in the configuration without safety gap produces greater overpressures in the far field than that in the $10 \mathrm{~m}$ safety gap configuration, while the opposite observation is seen when it comes to the simulation group case 9 to 16 , where the overpressures in the $10 \mathrm{~m}$ safety gap configuration become comparatively larger. It can be explained that the gaps between three adjacent modules (e.g. Module 3, Module 9 and Module 10) in North-South direction have different distance, which results in different flame turbulence interruption effects. For instance, it is seen in Fig. 14(a) that the flames propagating from ignition point in Module 10 towards to Module 3 firstly experience a small safety gap, then the flames are followed by a greater distance safety gap between 
Module 9 and Module 3, while if the flames start oppositely from ignition in Module 3 towards to Module 10, different turbulence path order could be seen.

Unlike the flame path 1 simulations, the flame path 2 experiences the same safety gaps arrangement order regardless of the flame propagation direction in North-South, which is due to the geometrical symmetry of the cylindrical layout. Therefore, from case 17 to 24 , the same overwhelming tendency is observed, namely, the far field overpressures without safety gap configuration exceeds the overpressures in the $10 \mathrm{~m}$ safety gap counterpart.

Overall, the arrangement order and the distance of the safety gap between the modules play critical roles. In order to quantify the safety gap distance and investigate its effect systematically, the following two sets of artificial configurations are modelled.

\subsubsection{Safety gaps between three congested regions}

The artificial configurations with three congestions are firstly modelled to investigate the corresponding explosion scenario in Section 3.2 where the flame path 1 goes through two safety gaps (Fig. 16). The FLACS models are mimicking tests extracted from the Research to Improve Guidance on Separation Distance for the Multi-energy Method (RIGOS)-research program [23], and the overpressure calculations for those artificial models had been validated in preview work [10]. 
In FLACS, those three modules in Fig. 16 are modelled with the same obstacle diameter of $19.1 \mathrm{~mm}$ and volume blockage ratios of $10.1 \%$, and all obstacles are orientated orthogonally and regularly. The ignition locations in this study are in the centre of the donor on the left hand side of the configuration.

8 simulation scenarios with 8 different safety gaps are set up, for all those simulations, the overall distance from the donor to Acceptor 2 is fixed, while Acceptor 1 is at varying locations in between the donor and Acceptor 2. Here, the authors define the safety gap distance ratio as the ratio of the Safety gap 1 distance divided by the distance of Safety gap 2. Overpressure monitors are positioned at regular distances along the axis of the donor-acceptor configurations, the safety gap distance ratio increases from 0.1 in case (1) to 4 in case (8), as seen in Fig. 17.

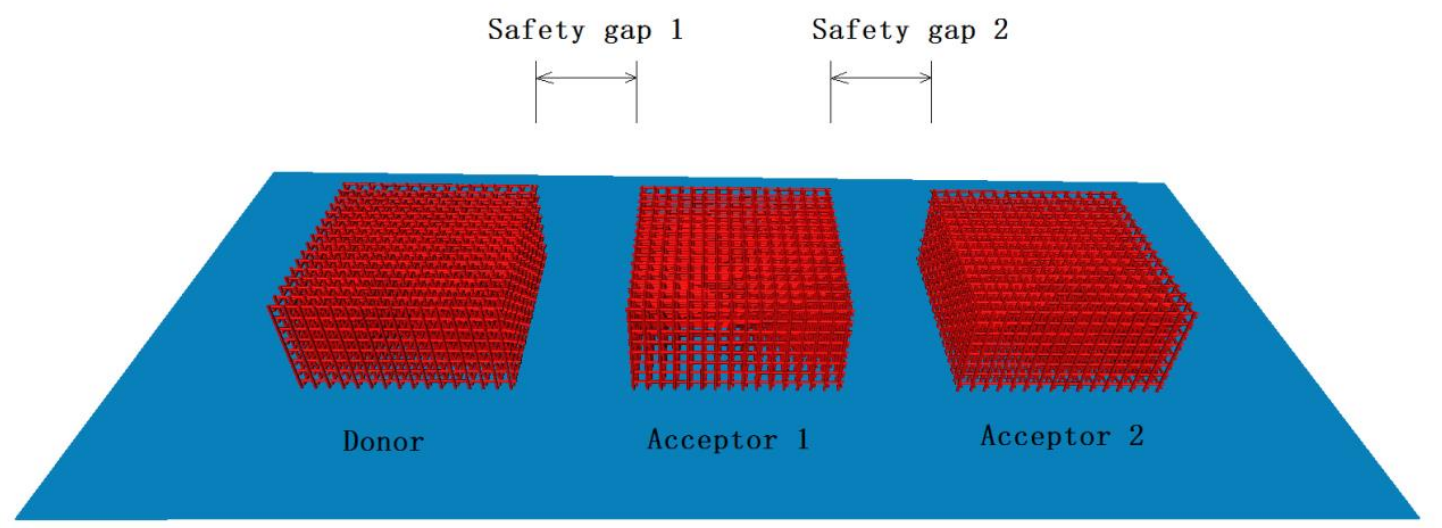

Fig. 16 Congested configurations with two safety gaps 


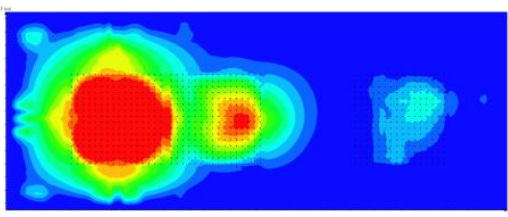

(1)

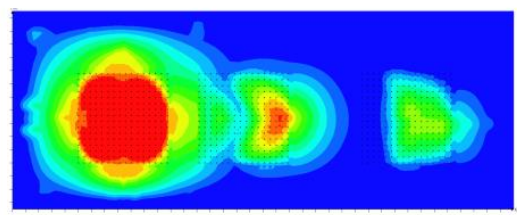

(3)

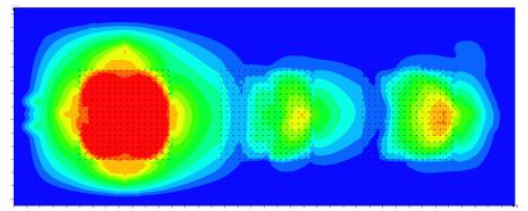

(5)

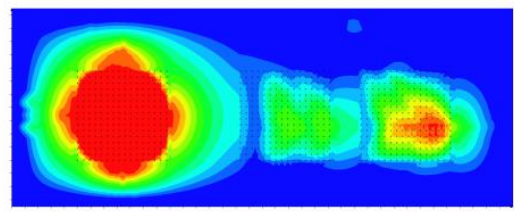

(7)

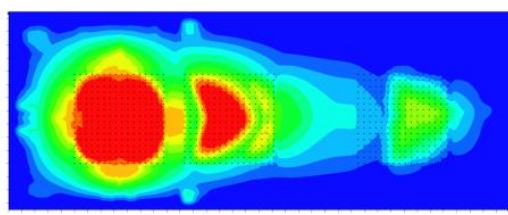

(2)

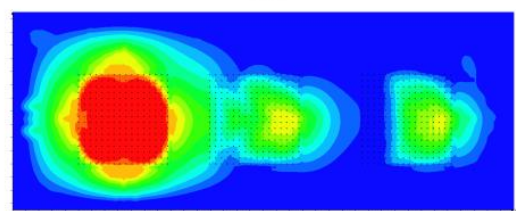

(4)

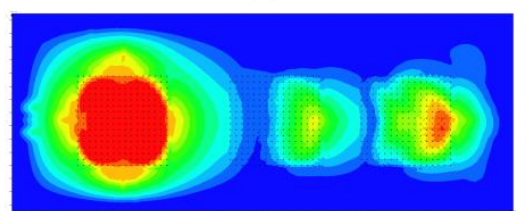

(6)

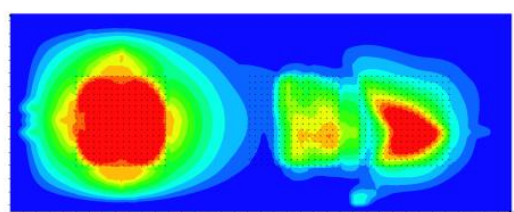

(8)
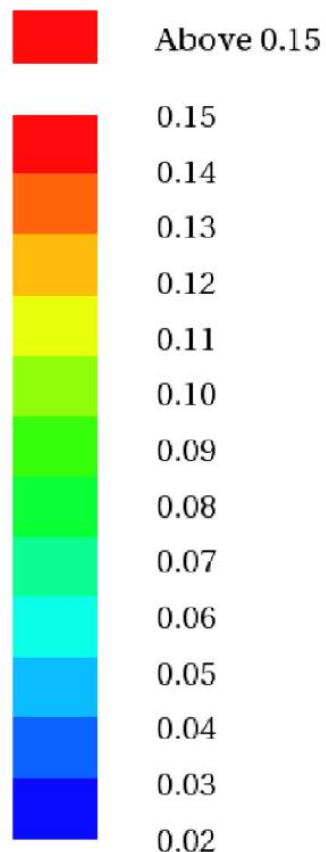

0.15

0.14

0.13

0.12

0.11

0.10

0.09

0.08

0.07

0.06

0.05

0.04

0.03

0.02

Below 0.02

Fig. 17 Overpressures in different configurations with two safety gaps (bar)

It is seen in Fig. 17 that the overpressures in the farthest module - Acceptor 2 increases when Acceptor 1 is placed increasingly closer to Acceptor 2, the maximum overpressures is observed in simulation case 8 which has the greatest safety gap distance ratio, the main reason is that the congestion volume in case 8 is increased once Acceptor 1 connects to Acceptor 2, and the larger congestion volume leads to longer flame turbulence path, thereby increasing the overpressure generation in Acceptor 2. So, if the target of protection is Acceptor 2 in the farthest end, the solution in such case is to maximize the distance of Safety gap 2 which can tremendously decelerate the flame turbulence in the open space, such as the example case 1 shown in Fig. 17. 
For each accepting module, the overpressures in the monitor points are averaged and recorded in Fig. 18. Unlike the overpressure increase in Acceptor 2, it is interesting to note that the averaged overpressure in Acceptor 1 decreases from case 1 to 7 , which is due to the fact that the increasing Safety gap 1 between the donor and Acceptor 1 amplifies the flame turbulence interruption effect.

Overall, depending on the overpressure safeguarding targets, different approaches are available in explosion mitigation. Firstly, in order to minimize the overpressure in Acceptor 2, the Safety gap 2 should have the greatest distance to discharge the flame turbulence and overpressure generation. Secondly, if the task is to safeguard Acceptor 1, sufficient distance of Safety gap 1 should be applied. Thirdly, if it is to balance the overpressures in Acceptor 1 and Acceptor 2, the optimal solution is to make the safety gap distance ratio equal to 1, namely Safety gap 1 and Safety gap 2 have the same distances, as seen the intersection point in Fig. 18. 


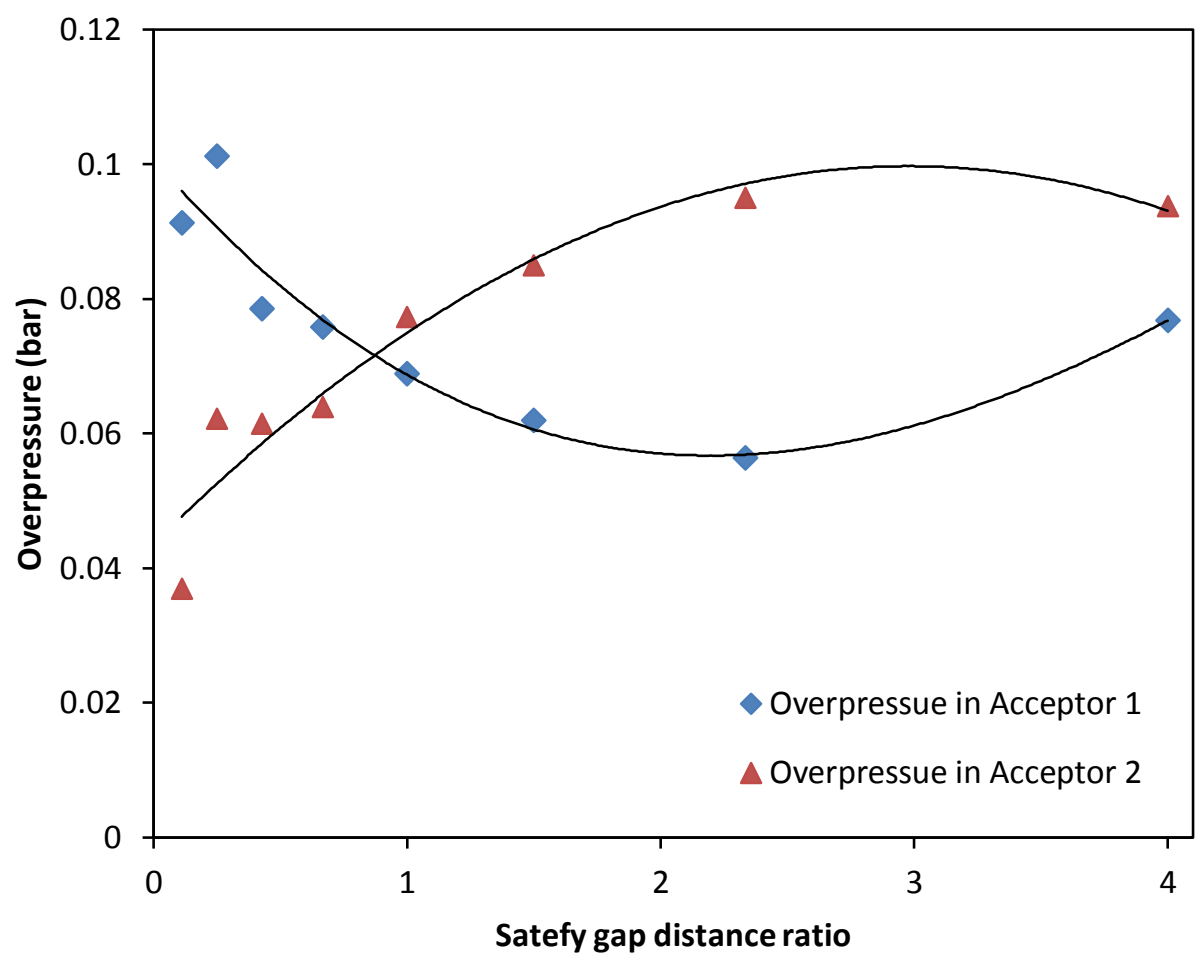

Fig. 18 Overpressures in accepting modules subjected to two safety gaps

\subsubsection{Safety gaps between four congested regions}

Furthermore, the second set of artificial configurations with four congestions is modeled to investigate the corresponding explosion scenario in Section 3.2 where the flame path 2 propagates through three safety gaps (Fig. 19). The obstacle diameter, arrangement and volume blockage ratio are same as they are in Section 3.2.1. The distance from the donor to Acceptor 2 is fixed, while the two congested regions in the middle move oppositely so that Safety gap 1 equals to Safety gap 3. As seen in Fig. 20, 8 simulation scenarios with 8 different safety gap distance ratios are modeled, the safety gap distance ratio is defined as the distance of Safety gap 1 divided by the distance of Safety gap 2. 


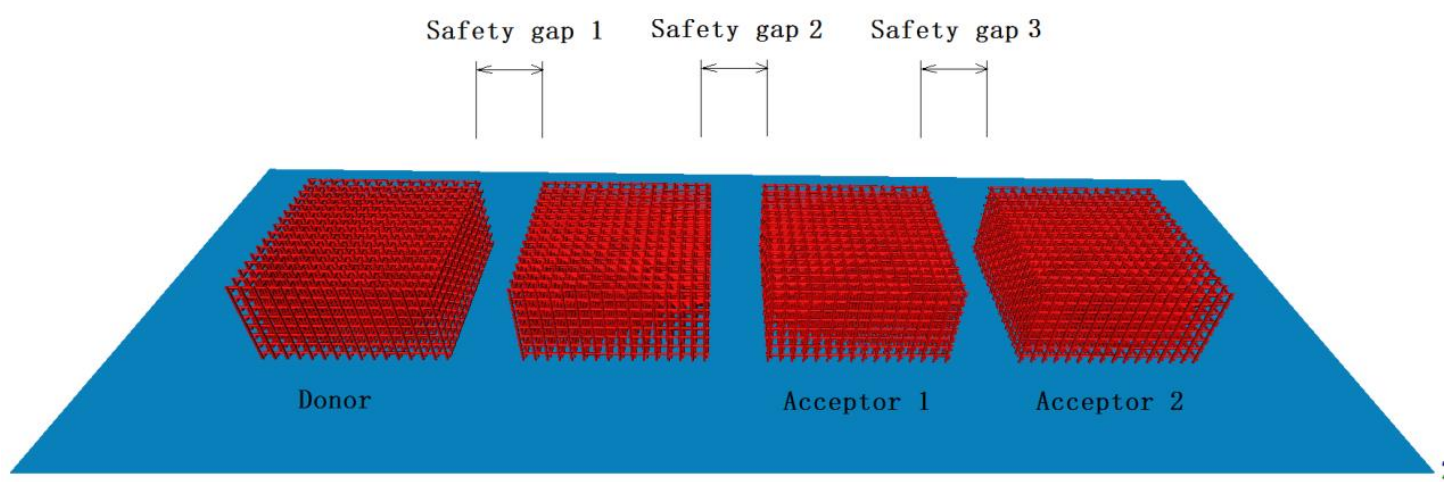

Fig. 19 Congested configurations with three safety gaps

As the simulation cases being conducted from (1) to (8) in Fig. 20, the overpressures in the three safety gap configuration have obvious decreasing tendency in Acceptor 2 compared to the overpressure varying tendencies in Fig. 17.

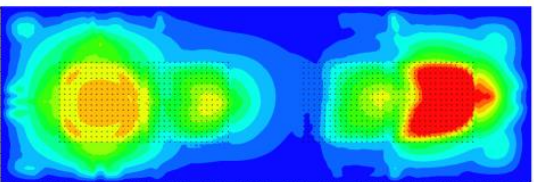

(1)

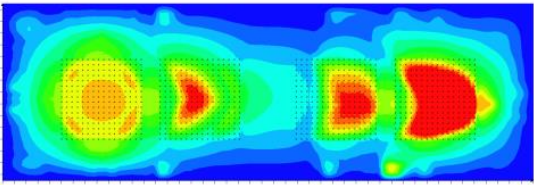

(3)

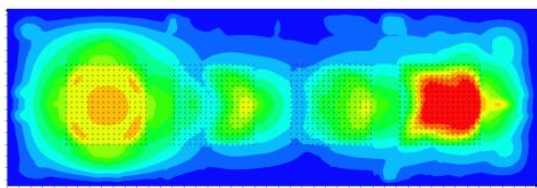

(5)

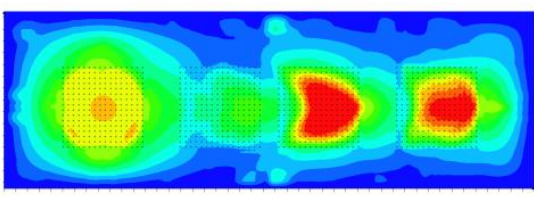

(7)

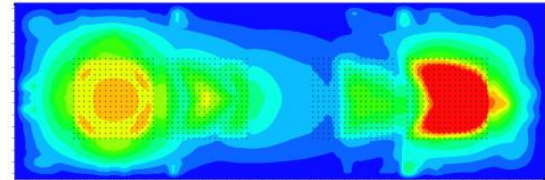

$(2)$

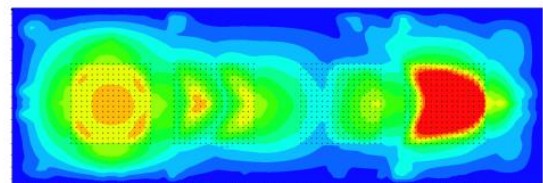

(4)

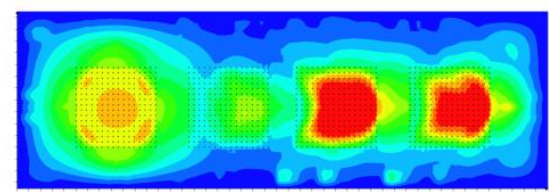

(6)

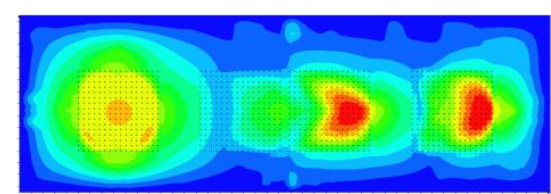

(8)

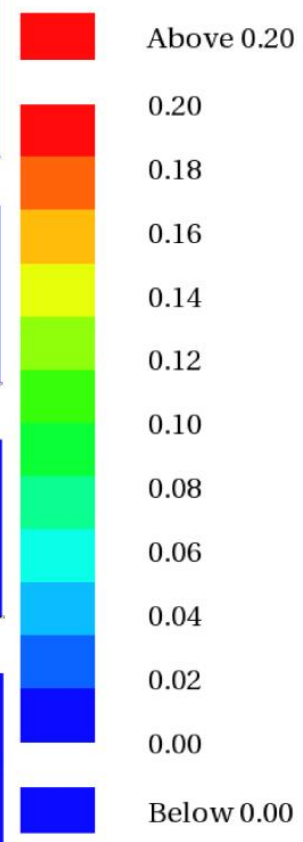

Below 0.00

Fig. 20 Overpressures in different configurations with three safety gaps (bar)

However, it is seen in Fig. 21 that the averaged overpressures in Acceptor 1 significantly increase along with the increase of the safety gap distance ratio from 
case 1 to 7 , which means that although the safety gaps 1 and 3 effectively reduce the overpressures in the farthest field (Acceptor 2) by manipulating the middle congestions, Acceptor 1 on the other hand is subjected to greater explosion overpressures. Therefore, depending on the protecting target under such circumstances, the arrangement of safety gaps provides different overpressure mitigation solutions. For example, the larger Safety gap 2 benefits Acceptor 1, such as case 1 , whereas the greater distance of Safety gap 1 and 3 significantly mitigate the explosion overpressures in Acceptor 2. The balanced overpressures in both accepting modules would exist at the intersection point in Fig. 21 where Safety gap 2 is about 1.5 times greater than Safety gap 1 or 3 , in such scenario the overpressures are alleviated in both Acceptor 1 and 2, as shown in the example - case 5.

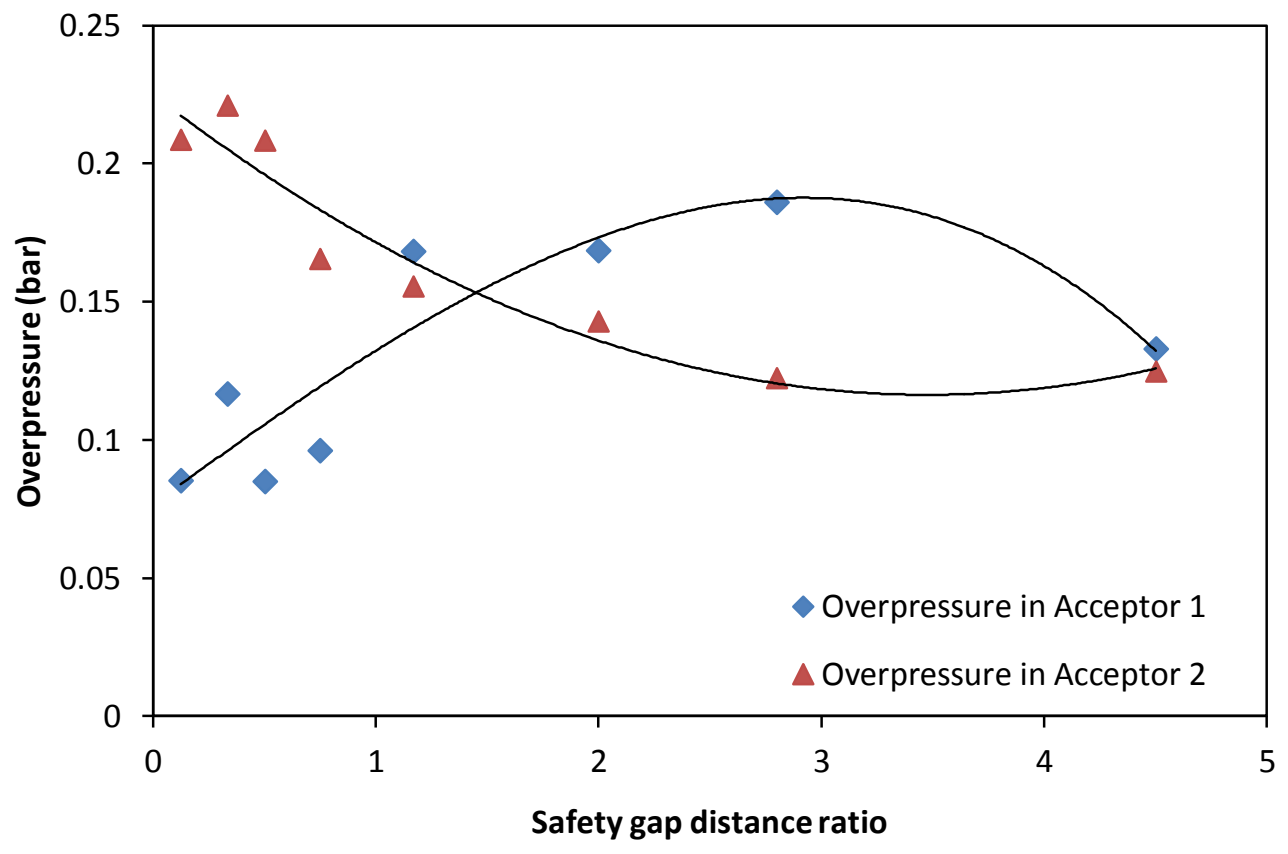

Fig. 21 Overpressures in accepting modules subjected to three safety gaps 


\subsubsection{Discussion}

The investigation to the two sets of artificial configurations well illustrates the overpressure distribution phenomenon on the cylindrical FLNG platform in Section 3.2.

For the simulations of the artificial configurations with two safety gaps, in case 1 to 8 of Table 2, the cylindrical FLNG platform without safety gap is the equivalent scenario of case 8 in Fig. 17, while the 10m safety gap cylindrical FLNG platform is the equivalent scenario of case 7 . In those scenarios, the gas cloud is ignited in the donor (i.e. equivalent Module 3 on the cylindrical FLNG platform), Acceptor 1 and 2 are equivalent Module 9 and 10. It is seen in Fig. 17 that if Acceptor 1 closely connects to Acceptor 2 in case 8, the overpressures in Acceptor 2 become greater than that in case 7. In other words, more safety gap space against Acceptor 2, as in case 7, reduces overpressures in the accepting modules, which reflects the phenomenon that the far field overpressures are smaller on the cylindrical FLNG platform with $10 \mathrm{~m}$ safety gap.

However, for simulations from case 9 to 16 in Table 2, the overpressure distribution phenomenon on the cylindrical FLNG platforms can be explained by corresponding scenario 1 and 2 in Fig. 17.

Comparing scenario 2 to scenario 1 in Fig. 17, the closer distance between the donor and Acceptor 1 in scenario 1 generates smaller overpressures in the far end Acceptor 
2 due to the greater distance of Safety gap 2 . It is the equivalent scenario on the cylindrical FLNG platform where the ignition relocates to Module 10, but the far field overpressures on the cylindrical FLNG platform with 10m safety gap are greater.

In terms of simulation cases from 17 to 24 in Table 2, the flame propagates from the edge module through three safety gaps to the farthest module, which is the equivalent artificial model in Section 3.2.2. The cylindrical FLNG with safety gap of 10m equals to the simulation case 8 in Fig. 20 where the safety gaps against the donor and Acceptor 2 effectively interrupt the flame turbulence and further mitigate overpressures in the far field.

In summary, 16 different artificial configurations are numerically simulated with different ignition locations and gas cloud on the cylindrical FLNG platform. It is concluded that the $10 \mathrm{~m}$ safety gaps on the cylindrical FLNG platform effectively mitigate overpressures in the far field modules in most cases. However, the exception is seen in some scenarios that the overpressures are increased in the far field due to the flame turbulence interaction with the adjacent modules. Therefore, the solution to balance overpressures in all far field modules, is to achieve the balancing safety gap distance ratio with the safety gap that plays the most efficient role in overpressure mitigation. 


\section{Conclusion}

As an extension of the previous paper [1], regarding the gas dispersion simulations on a cylindrical Floating Liquefied Natural Gas (FLNG) platform, the same cylindrical FLNG is utilized in this study to further investigate the gas explosion overpressure mitigating effect of safety gap on the congested and confined offshore configurations. The gas explosion simulations are conducted by using the commercial CFD software FLACS, (and) both near field and far field explosion scenarios are investigated.

The Data-dump technique which improves the FLACS overpressure calculation is applied. The overall results indicate that the larger the safety gap, the more efficient is the Data-dump technique to correct overpressure prediction. Therefore, the Datadump is utilized throughout this paper to ensure a more reliable gas explosion overpressure calculation for all scenarios with safety gaps.

In the near field gas explosion simulations, overpressure mitigation phenomenon is observed by applying the safety gap into the congested regions. It is concluded that the increase of safety gap size results in greater overpressure reduction in the accepting modules, and the safety gap reduces more overpressures where the average obstacle diameter is smaller, the flame path is longer and the congestion ratio is greater.

In terms of the far field gas explosion, the simulations are conducted on two cylindrical FLNG configurations with and without 10m safety gap in North-South 
direction. For each FLNG configuration, 24 simulations with 4 different gas clouds and 6 different ignition locations are carried out. The corresponding artificial models well demonstrate that depending on the ignition and the overpressure mitigation target locations, the safety gaps play different roles in reducing overpressures. Overall, in order to optimize the gas explosion alleviation effect of safety gap on the cylindrical FLNG platform, the most effective way is to balance overpressures in all far field modules and to achieve the balancing safety gap distance ratio accordingly. 


\section{References}

1. Li, J.D., et al., Gas dispersion risk analysis of safety gap effect on the innovating FLNG vessel with a cylindrical platform. Journal of Loss Prevention in the Process Industries, 2016. 40: p. 304-316.

2. Zhao, W.H., et al., Recent developments on the hydrodynamics of floating liquid natural gas (FLNG). Ocean Engineering, 2011. 38(14-15): p. 15551567.

3. Zhao, W.H., et al., Hydrodynamics of an FLNG system in tandem offloading operation. Ocean Engineering, 2013. 57: p. 150-162.

4. Shimamura, Y., FPSO/FSO: State of the art. Journal of Marine Science and Technology, 2002. 7(2): p. 59-70.

5. Suardin, J.A., et al., Fire and explosion assessment on oil and gas floating production storage offloading (FPSO): An effective screening and comparison tool. Process Safety and Environmental Protection, 2009. 87(3): p. 147-160.

6. Hetland, J., et al., Integrating a full carbon capture scheme onto a $450 \mathrm{MWe}$ NGCC electric power generation hub for offshore operations: Presenting the Sevan GTW concept. Applied Energy, 2009. 86(11): p. 2298-2307. 
7. Wang, F., et al., Floating non-traditional manufacture of floating drilling storage and offloading units-study on modeling and optimization method for the underwater rotating technology. Marine Structures, 2013. 31: p. 15-23.

8. Kvamsdal, H.M., et al., Maintaining a neutral water balance in a $450 \mathrm{MWe}$ NGCC-CCS power system with post-combustion carbon dioxide capture aimed at offshore operation. International Journal of Greenhouse Gas Control, 2010. 4(4): p. 613-622.

9. Hirdaris, S.E., et al., Loads for use in the design of ships and offshore structures. Ocean Engineering, 2014. 78: p. 131-174.

10. Ma, G., J. Li, and M. Abdel-jawad, Accuracy improvement in evaluation of gas explosion overpressures in congestions with safety gaps. Journal of Loss Prevention in the Process Industries, 2014. 32: p. 358-366.

11. GexCon, FLACS v9.1 User's Manual. 2011, Norway: Doxygen.

12. Bleyer, A., et al., Comparison between FLACS explosion simulations and experiments conducted in a PWR Steam Generator casemate scale down with hydrogen gradients. Nuclear Engineering and Design, 2012. 245: p. 189-196.

13. Hansen, O.R., et al., Validation of FLACS against experimental data sets from the model evaluation database for LNG vapor dispersion. Journal of Loss Prevention in the Process Industries, 2010. 23(6): p. 857-877. 
14. Middha, P., et al., CFD calculations of gas leak dispersion and subsequent gas explosions: Validation against ignited impinging hydrogen jet experiments. Journal of Hazardous Materials, 2010. 179(1-3): p. 84-94.

15. Middha, P., O.R. Hansen, and I.E. Storvik, Validation of CFD-model for hydrogen dispersion. Journal of Loss Prevention in the Process Industries, 2009. 22(6): p. 1034-1038.

16. Pwaga, S.S., Sensitivity Analysis of Proposed LNG liquefaction Processes for LNG FPSO, in Department of Energy and Process Engineering. 2011, Norwegian University of Science and Technology: Norway.

17. $\mathrm{Xu}, \mathrm{X} . \mathrm{W}$., et al., The correlation between mixed refrigerant composition and ambient conditions in the PRICO LNG process. Applied Energy, 2013. 102: p. 1127-1136.

18. $\mathrm{Xu}, \mathrm{X} . \mathrm{W}$., J.P. Liu, and L. Cao, Optimization and analysis of mixed refrigerant. composition for the PRICO natural gas liquefaction process. Cryogenics, 2014. 59: p. 60-69.

19. Li, J., M. Abdel-jawad, and G. Ma, New correlation for vapor cloud explosion overpressure calculation at Congested Configurations. Journal of Loss Prevention in the Process Industries, 2014. 31: p. 16-25.

20. Li, J., G. Ma, and M. Abdel-jawad, Evaluation of gas explosion overpressures at configurations with irregularly arranged obstacles. Journal of Performance of Constructed Facilities, 2014. 
21. van den Berg, A.C. and A.L. Mos, Research to improve guidance on separation distance for the multi-energy method (RIGOS). TNO Prins Maurits Laboratory, 2005. RESEARCH REPORT 369.

22. Bradley, D., M. Lawes, and K.X. Liu, Turbulent flame speeds in ducts and the deflagration/detonation transition. Combustion and Flame, 2008. 154(1-2): p. 96-108.

23. van den Berg, A.C. and N.H.A. Versloot, The multi-energy critical separation distance. Journal of Loss Prevention in the Process Industries, 2003. 16(2): p. 111-120. 